\title{
Contingency tables by bipartite graphs and intersection graphs
}

\author{
S.AbdElrahman(3) El-Zohny H(1),M.M. Khalil(2) \\ ${ }^{1,2,3}$ Mathematics Department, Faculty of scienceAlAzhar UniversityCairo- Egypt
}

\begin{abstract}
In this paper we will convert weighted bipartite graph to intersection graph. Also we introduce the variation of the algorithm of bipartite graph and intersection graph.

Keywords:Graph, Contingency table, bipartite graph, intersection graph, clique, algorithms.
\end{abstract}

\section{Definitions:-}

\section{Introduction}

Abstract graph:

A graph $\mathrm{G}=(\mathrm{V}, \mathrm{E})$ consists of a set of vertices $\mathrm{V}$ and a set of edges E. Elements of $\mathrm{V}$ are distinct. Elements of $\mathrm{E}$ are 2- sets of the form $\{\mathrm{x}, \mathrm{y}\}$, where $\mathrm{x}$ and $\mathrm{y}$ are both vertices in $\mathrm{V}$.

\section{Contingency table:}

A simple 2 way Contingency table: is a common method of analyzing the association between two categorical(qualitative) variables.

A p-way contingency table when $(\mathrm{p}>2)$.

\section{Bipartite graph:}

A graph $G=(V, E)$ is called bipartite where $V=X \cup Y$, with $X \cap Y=\varnothing$, and every edge of $G$ of the form $\{a, b\}$ with $a \in X, b \in Y$.

\section{Intersection graph:}

A graph $G=(V, E)$ is called intersection graph where the set of vertices is formed by a family of sets. Given two vertices $a$ and $b$ there is an edge $\{a, b\}$ in $E$ iffa $\cap b \neq \varnothing$.

Both Bipartite graph and intersection graph has no loops or multiple edges.

\section{Multivariate data analysis:}

Data with more than one variable

\section{Clique:}

defined to be a sub graph in which each pair of vertices joined by edge.

\section{Algorithm:}

In mathematics, computer science, and related subjects, an algorithm is a finite sequence of steps expressed for solving a problem. Algorithm can be expressed as

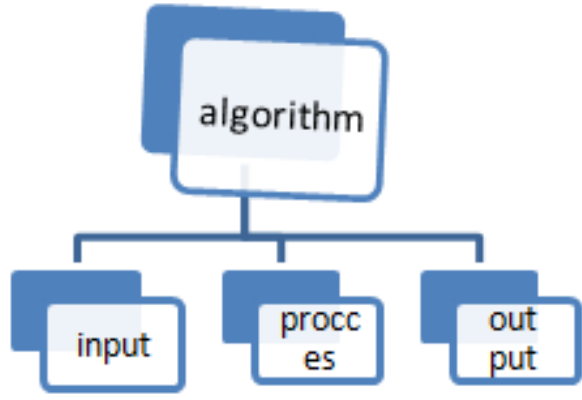

\section{Main results}

We use graph theory to read the data given in contingency table, and cluster it .by this way the data converted to weighted bipartite graph and weighted intersectiongraph. 
First:- For bipartite graph

Example (1)

Consider the simple contingency table (1) for 50 metal to detect specularity according to their size

\begin{tabular}{|c|c|c|}
\hline & small & large \\
\hline Specular & 4 & 13 \\
\hline Non specular & 15 & 18 \\
\hline
\end{tabular}

Table (1)

We can convert this table to weighted bipartite graph, where the weight on the edges corresponding to the frequencies of the cell in the table.

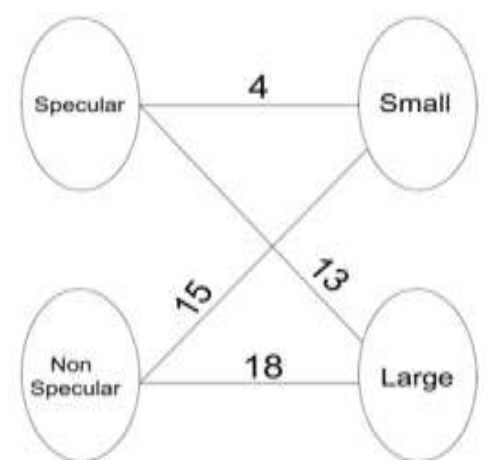

Figure (1) Weighted Bipartite graph

\section{Example (2)}

Consider the data base for different animals shown in the table (2):

\begin{tabular}{|c|c|c|c|c|c|c|c|c|c|}
\hline & & $\begin{array}{l}\text { छ्ञ } \\
\text { चี }\end{array}$ & 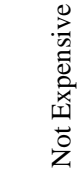 & 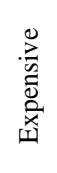 & 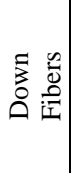 & 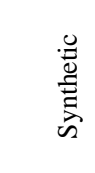 & $\begin{array}{l}\overline{8} \\
8 \\
0\end{array}$ & $\begin{array}{l}\frac{0}{0} \\
\frac{\pi}{0} \\
\frac{0}{0} \\
\frac{0}{<}\end{array}$ & Iี \\
\hline & Sleeping Bag & \multicolumn{3}{|c|}{ Price } & \multicolumn{2}{|r|}{ Fiber } & \multicolumn{3}{|c|}{ Quality } \\
\hline 1 & One kilo Bag & 1 & 0 & 0 & 0 & 1 & 1 & 0 & 0 \\
\hline 2 & Sund & 1 & 0 & 0 & 0 & 1 & 0 & 0 & 1 \\
\hline 3 & Kompack Basic & 1 & 0 & 0 & 0 & 1 & 1 & 0 & 0 \\
\hline 4 & FinmarkTour & 1 & 0 & 0 & 0 & 1 & 0 & 0 & 1 \\
\hline 5 & InterlightLyx & 1 & 0 & 0 & 0 & 1 & 0 & 0 & 1 \\
\hline 6 & Kompact & 0 & 1 & 0 & 0 & 1 & 0 & 1 & 0 \\
\hline 7 & Touch the Cloud & 0 & 1 & 0 & 0 & 1 & 0 & 1 & 0 \\
\hline 8 & Cats Meow & 0 & 1 & 0 & 0 & 1 & 1 & 0 & 0 \\
\hline 9 & Igloo Super & 0 & 1 & 0 & 0 & 1 & 0 & 0 & 1 \\
\hline 10 & Donna & 0 & 1 & 0 & 0 & 1 & 0 & 1 & 0 \\
\hline 11 & Tyin & 0 & 1 & 0 & 0 & 1 & 0 & 1 & 0 \\
\hline 12 & Travellers Dream & 0 & 1 & 0 & 1 & 0 & 1 & 0 & 0 \\
\hline 13 & Yeti Light & 0 & 1 & 0 & 1 & 0 & 1 & 0 & 0 \\
\hline 14 & Climber & 0 & 1 & 0 & 1 & 0 & 0 & 1 & 0 \\
\hline 15 & Viking & 0 & 1 & 0 & 1 & 0 & 1 & 0 & 0 \\
\hline 16 & Eiger & 0 & 0 & 1 & 1 & 0 & 0 & 1 & 0 \\
\hline 17 & Climber Light & 0 & 1 & 0 & 1 & 0 & 1 & 0 & 0 \\
\hline 18 & Cobra & 0 & 0 & 1 & 1 & 0 & 1 & 0 & 0 \\
\hline 19 & Cobra Comfort & 0 & 1 & 0 & 1 & 0 & 0 & 1 & 0 \\
\hline 20 & Foxfire & 0 & 0 & 1 & 1 & 0 & 1 & 0 & 0 \\
\hline 21 & Mont Blanc & 0 & 0 & 1 & 1 & 0 & 1 & 0 & 0 \\
\hline
\end{tabular}




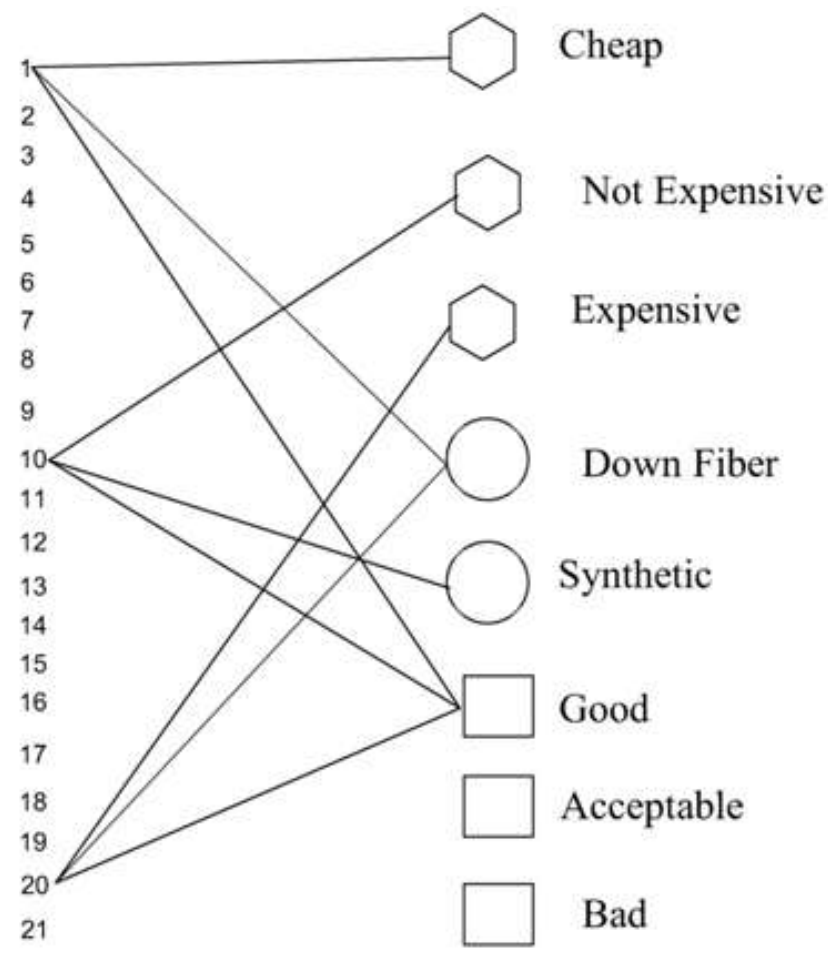

Bipartite graph due to the data from conengency table

Figure (2)

\section{Second:- For intersection graph:}

The difference between intersection graphs and bipartite graphs is the vertices where in the case of intersection graphs the vertices are sets.

We can convert weighted bipartite graph in example (1) into weighted intersection graph by put specular (1yes, 0 -no), and size(1-small,0-large).as shown in the figure (3)

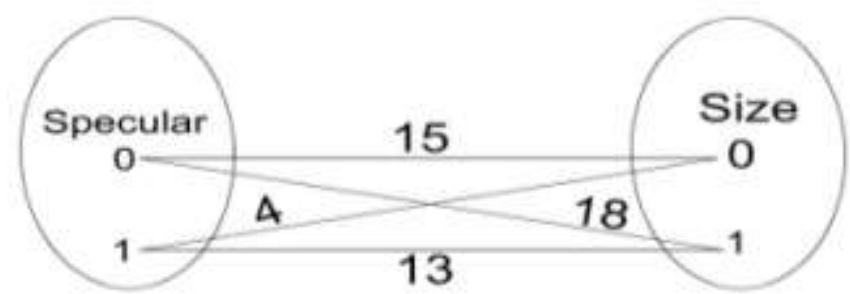

Figure (3)Weighted Intersection graph

By using intersection graphs the observations can be read as clique [if we have 3variables we get 3 clique, and if we have 4 variable we get 4 clique].

Example :suppose we observe3 characteristic of chicken $\{$ Fins $(0,1)$,domestic $(0,1), \operatorname{legs}(0,2,4)\}$.and represent them in the traditional tabular form

Tabular form

\begin{tabular}{|c|c|c|c|}
\hline Chicken & 0 & 1 & 2 \\
\hline & Fins & Domestic & Legs \\
\hline
\end{tabular}

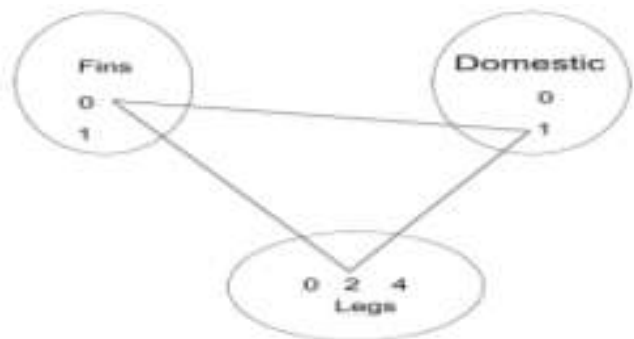

3-Clique

Figure (4) 
Example (3)

Consider sample fromzoo data set that consist of 17 characteristic of 101 animals. For simplicity we selected a subset of these sample consist of 15 animals and 4 variables. The animals are
1) Antelope
2)bear
3)carp4)catfish
5) chicken

6) clam7)crow8)duck9)goat10)sealion

11) worm12)elephant13)cat 14)monkey15)bat

and the variables are $\{\operatorname{wild}(0,1), \operatorname{Fins}(0,1)$, domestic $(0,1), \operatorname{legs}(0,2,4)\}$.

Simple 2- way contingency table:

We have two variables (Domestic, Legs) to convert contingency table into intersection graph

\begin{tabular}{|c|c|c|c|c|}
\hline & \multicolumn{3}{|c|}{ Legs } & Total \\
\hline & 0 & 2 & 4 & \\
\hline Domestic 0 & 3 & 4 & 4 & 11 \\
\hline Domestic 1 & 1 & 1 & 2 & 4 \\
\hline Total & 4 & 5 & 6 & 15 \\
\hline
\end{tabular}

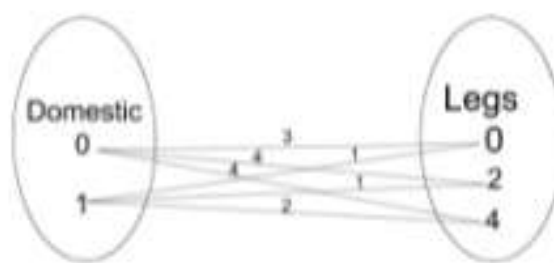

\section{Weighted intersection graph}

An algorithm to convert 2-way contingency tables into intersection graphs:-

[1] Start

[2] read the data sample $\mathrm{Xi}, \mathrm{j}, \mathrm{Wi}, \mathrm{j}$ (where i rows, and $\mathrm{j}$ columns , Wi,j is the frequency between $\mathrm{i}, \mathrm{j}$ )

[3] For $(\mathrm{i}=0, \mathrm{i}<=1, \mathrm{i}++)$

[4] For $(j=0, j<=4, j+2)$

[5] If $\exists$ relation between $i, j$ draw an edge between $i, j$

[6] PrintWi,j on the edge

[7] Else go to line 3

[8] End

Consider the table

\begin{tabular}{|l|l|l|l|l|l|}
\hline & & Fins & Domestic & Legs & Wild \\
\hline 1 & Antelope & 0 & 0 & 4 & 1 \\
\hline 2 & Bear & 0 & 0 & 4 & 1 \\
\hline 3 & Carp & 1 & 1 & 0 & 0 \\
\hline 4 & Catfish & 1 & 0 & 0 & 0 \\
\hline 5 & Chicken & 0 & 1 & 2 & 1 \\
\hline 6 & Clam & 0 & 0 & 0 & 0 \\
\hline 7 & Crow & 0 & 0 & 2 & 1 \\
\hline 8 & Duck & 0 & 0 & 2 & 1 \\
\hline 9 & Goat & 0 & 1 & 4 & 1 \\
\hline 10 & Sea lion & 1 & 0 & 2 & 0 \\
\hline 11 & Worm & 0 & 0 & 0 & 1 \\
\hline 12 & Elephant & 0 & 0 & 4 & 1 \\
\hline 13 & Cat & 0 & 1 & 4 & 1 \\
\hline 14 & Monkey & 0 & 0 & 4 & 1 \\
\hline 15 & Bat & 0 & 0 & 2 & 1 \\
\hline
\end{tabular}

The data in the table converted to intersection graph where the vertices of the graph are the variables. 


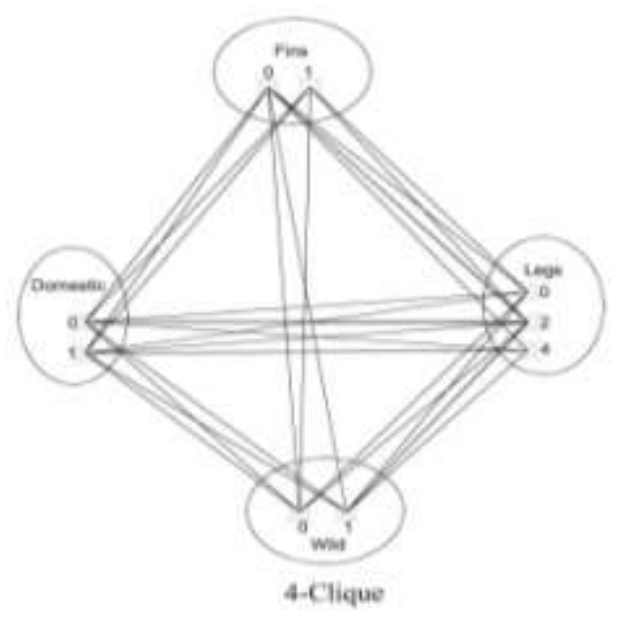

\section{Conditional algorithm for clusters intersection graphs:}

[1] Start

[2] Read the data base $\mathrm{Xi}, \mathrm{j}, \mathrm{k}, \mathrm{l}$ with $\mathrm{Wi}, \mathrm{j}, \mathrm{k}, \mathrm{l}$

[3] Draw graph with nodes i,j,k,l

[4] For $(\mathrm{i}=0, \mathrm{i}<=1, \mathrm{i}++)$

[5] For $(j=0, j<=1, j++)$

[6] For $(\mathrm{k}=0, \mathrm{k}<=4, \mathrm{k}+2)$

[7] For $(\mathrm{l}=0,1<=1,1++)$

[8] If the relation between $\mathrm{I}, \mathrm{j}, \mathrm{k}, \mathrm{l}$ occurs twice or more draw the relations

[9] Else

[10] Go to line 4

[11] End

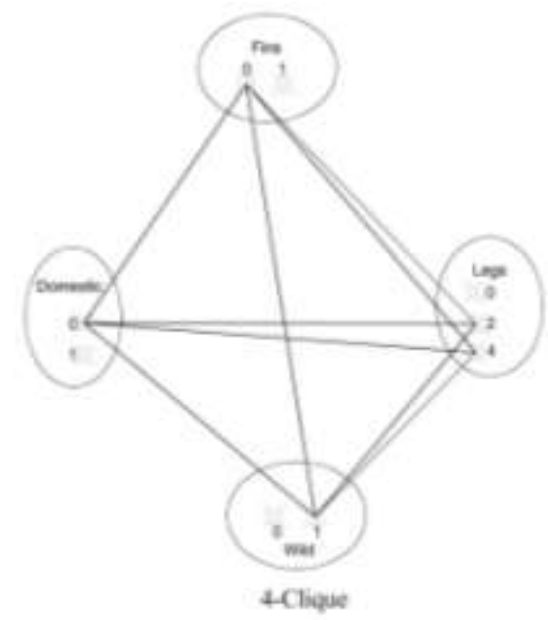

References

[1]. Cook ,D.J.,Holder,L.,B.,2006.Mining graph data. New York :john.

[2]. Intersection graph - based representation of contingency tables, Valter Martins Vairinhos.

[3]. Anderson,T.W.(1984).An introduction to multivariate statistical Analysis,John Wiley.

[4]. Intersectiongraph :an introduction ,Madhumangal Pal 2013.

[5]. SatuElisa,:graph clustering, Computer Science Review, (2007),27-64. 